\title{
A model for removing sediments from open channels
}

\author{
Hassan A. Omran ${ }^{1}$, Mohammed Y. Fattah ${ }^{1 *}$ and Sadiq Q. Sulaiman ${ }^{2}$ \\ ${ }^{1}$ Building and Construction Engineering Department, University of Technology, Baghdad, Iraq. \\ ${ }^{2}$ Civil Engineering Department, University of Al-Anbar, Iraq.
}

Received 18 November, 2013; Accepted 14 February, 2014

\begin{abstract}
The problems caused by suspended sediment overloading in open channels are of great importance to the hydraulic engineers. One of the important problems caused by the sediment overloading during flood seasons is the change in bed level and corresponding water level of rivers due to its deposition on the river bed. At the upstream reaches, the bed slope is high, velocity is high and hence the rivers carry large amount of sediment during flood seasons. In this paper, eighty-one experiments on a laboratory open-channel of cross-section dimensions $\left(20^{\star} 50\right) \mathrm{cm}$ and $(10) \mathrm{m}$ long. The experiments include using sharp crested weir in the last third part of the channel. Three heights of weir are used; namely half width of channel (B), equal to the width, and 1.5 times the width of channel). Also, three bed slopes of the channel are used; namely $0.015,0.0225$, and 0.03 ). In all experiments, the values of discharge are limited between 100 and $300 \mathrm{l} / \mathrm{min}$. The concentration of suspended sediment is measured upstream and downstream the weir through each experiment to calculate the sediment reduction ratio (SRR\%). The results of the laboratory experiments showed that the sediment reduction ratio increases with the increase of weir height and reaches about $(63 \%)$ at a channel bed slope of $(0.015)$ and a weir height $1.5 \mathrm{~B}$. When the discharge is more than $(100 \mathrm{I} / \mathrm{min})$, the highest values of sediment reduction ratio are obtained for the three slopes used and at weir height of 1.5B.
\end{abstract}

Key words: Open channel, sediment reduction ratio, model, removal.

\section{INTRODUCTION}

Many hydraulic engineering projects concern the control of sedimentation. For some large engineering projects, such as construction of a navigation channel over many kilometers in a shallow estuary, comprehensive control of sedimentation is extremely costly.

Slope-channel coupling and in-channel sediment storage can be important factors that influence sediment delivery through catchments. Sediment budgets offer an appropriate means to assess the role of these factors by quantifying the various components in the catchment sediment transfer system.
When a sediment beach covered by stones or an armor layer is exposed to breaking waves, the turbulence generated by the breaking waves can cause mobilization and removal of the sediment underneath the stones.

Sedimentation in dams, rivers, estuaries, and coastal regions has important environmental and economic influences. Different researchers studied the sediment transport and control in open channels. Zhu et al. (1999) presented an optimal-control model of sedimentation. The model contains the determination of optimal location and scheduling of dredging to minimize the total cost and

*Corresponding author. E-mail: myf_1968@yahoo.com.

Author(s) agree that this article remain permanently open access under the terms of the Creative Commons Attribution License 4.0 International License 
to obtain a channel where water depths are not less than specified values. The system governing equations which were used in the model are:

$$
\begin{aligned}
& \frac{\partial \vec{v}}{\partial t}+\vec{v} \cdot \nabla v+f(k \cdot \vec{v})=-g \cdot \nabla z+A \cdot \nabla \vec{v}-C_{D} \frac{\vec{v}}{h}---- \\
& \frac{\partial h}{\partial t}+\nabla(\vec{v} \cdot h)=0-------- \\
& \frac{\partial h \cdot S}{\partial t}+\nabla(\vec{v} h \cdot S)=\zeta \nabla(h \nabla S)+\alpha w\left(S^{\prime}-S\right)----
\end{aligned}
$$

where: $\vec{v}=$ Depth-averaged velocity in continuous governing equation, $k=$ Empirical constant, $h=$ Water depth in governing continuous equations, $S=$ Sediment mass mixed rate in continuous governing equations, $S^{\prime}=$ Sediment carrying ability of water flow, $t=$ Time step, $C_{D}=$ Drag coefficient, $\zeta=$ Diffusion coefficient of sediment, $w=$ Falling speed of sediment particle, and $\alpha=$ Empirical coefficient.

Barkdoll and Ettema (1999) determined the limits to which submerged vanes can be used in preventing excessive bed-sediment ingestion into lateral diversions of flow from alluvial channels, by a laboratory flume experiments. The experiments showed that a scheme of submerged vanes placed at the diversion entrance admits only a negligible rate of bed-sediment entry into the diversion when the ratio of unit discharge in the diversion to that in the main channel is less than about 0.2 . Beyond this value, the effectiveness of the vanes diminishes.

Nino (2002) developed a simple theory to account for the observed downstream variation of the median sediment size in Chilean rivers based on a reach-wise equilibrium sediment transport concept. The theory make use of a bed load transport equation, linked with a resistance equation to estimate the median sediment diameter as a function of channel slope with the flow Reynolds number and bed load concentration as parameters. The formulas which were used in this research are Meyer-Peter, Muller's, Ackers and White's formulas:

$$
\frac{q_{s}}{\sqrt{g R d_{s}^{3}}}=8\left(\tau_{*}-\tau_{*_{c}}\right)^{3 / 2}-------
$$

where: $q_{s}=$ Bed load transport rate, $R=$ Sediment submerge specific density, $d_{s}=$ Sediment size, $\tau_{*}=$ Dimensionless bed shear stress, $\tau_{*_{c}}=$ Threshold dimensionless value of bed shear stress, and $g=$ Acceleration of gravity.
The resulting model was validated against field data corresponding to 150 rivers in Central Chile, covering slopes in the range of 0.04 to $8.61 \%$ with median sediment size in the range of 0.3 to $250 \mathrm{~mm}$.

\section{Theoretical analysis}

The erosion, deposition, and transport of sediment by water arise in a variety of situations with engineering implications. Erosion must be considered in the design of stable channels or the design for local scour around bridge piers. Re-suspension of possibly contaminated bottom sediments has consequences for water quality. Deposition is often undesirable since it may hinder the operation, or shorten the working life, of hydraulic structures or navigational channels. Sediment traps are specifically designed to promote the deposition of suspended material to minimize their downstream impact, e.g., on cooling water inlet works, or in water treatment plants. A large literature exists on approaches to problems involving sediment transport; the following can only introduce the basic concepts in summary fashion.

It is oriented primarily to applications in steady uniform flows in a sand-bed channel; problems involving flow non-uniformity, unsteadiness, and gravel-beds, are only briefly mentioned and coastal processes are treated in the section on coastal engineering. Cohesive sediments for which physic-chemical attractive forces may lead to the aggregation of particles are not considered at all. The finer fractions (clays and silts) that are susceptible to aggregation are found more in estuarial and coastal shelf regions rather than in streams (Lyn, 2003).

\section{The characteristics of sediment}

\section{Density, size, and shape}

The exact shape of a sediment particle is not spherical, and so a compact specification of its geometry or size is not feasible. Two practical measures of grain size are: (i) the sedimentation or aerodynamic diameter - the diameter of the sphere of the same material with the same fall velocity, ws, under the same conditions, and (ii) the sieve diameter - the length of a side of the square sieve opening through which the particle will just pass. Because size determination is most often performed with sieves, the available data for sediment size usually refer to the sieve diameter, which is taken to be the geometric mean of the adjacent sieve meshes, that is, the mesh size through which the particle has passed, and the mesh size at which the particle is retained. The sedimentation diameter is related empirically to the sieve diameter by means of a shape factor, S.F., which increases from 0 to 1 as the particle becomes more spherical (for well-worn sand, S.F. $\approx 0.7$ ). 


\section{Size distribution}

Naturally occurring sediment samples exhibit a range of grain diameters. A characteristic diameter, da, may be defined in terms of the percent, a by weight of the sample that is smaller than $d a$. Thus, for a sample with $d_{84}=0.35$ $\mathrm{mm}, 84 \%$ by weight of the sample is less than $0.35 \mathrm{~mm}$ in diameter. The median size is denoted as $d_{50}$.

\section{Fall (or settling) velocity}

The terminal velocity of a particle falling alone through a stagnant fluid of infinite extent is called its fall or settling velocity, $\mathrm{w}_{\mathrm{s}}$. The standard drag curve for a spherical particle provides a relationship between $d$ and $w$.

\section{Angle of repose}

The angle of repose of a sediment particle is important in describing the initiation of its motion and hence sediment erosion of an inclined surface, such as a stream bank. It is defined as the angle, q, at which the particle is just in equilibrium with respect to sliding due to gravitational forces.

\section{Sediment transport}

Three modes of sediment transport are distinguished: wash load, suspended load, and bed load. Wash load refers to very fine suspended material, e.g., silt, that because of their very small fall velocities, interacts little with the bed. It will not be further considered since it is determined by upstream supply conditions rather than by local hydraulic parameters. Suspended load refers to material that is transported downstream primarily in suspension far from the bed, but which because of sedimentation and turbulent mixing still interacts significantly with the bed. Finally, bed load refers to material that remains generally close to the bed in the bedload region, being transported mainly through rolling or in short hops (termed saltation). The relative importance of the two modes of sediment transport may be roughly inferred from the ratio of settling velocity to shear velocity, $w_{s} / u^{*}$. For $w_{s} / u^{*}<0.5$, suspended load transport is likely dominant, while for $w_{s} / u^{*}>1.5$, bedload transport is likely dominant.

\section{Measurement of sediment transport}

In addition to, and contributing to, the difficulties in describing and predicting accurately sediment transport, total load measurements, particularly in the field are associated with much uncertainty. Natural alluvial channels may exhibit a high degree of spatial and temporal nonuniformities, which are not specifically considered in the 'averaged' models discussed above.

Standard methods of suspended load measurements in streams include the use of depth-integrating samplers that collect a continuous sample as they are lowered at a constant rate (depending on stream velocity) into the stream, and the use of point integrating samplers that incorporate a valve mechanism to restrict sampling, if desired, to selected points or intervals in the water column. Such sampling assumes that the sampler is aligned with a dominant flow direction, and that the velocity at the sampler intake is equal to the stream velocity.

In the vicinity of a dune-covered bed, these conditions cannot be fulfilled. The finite size of the suspended load samplers implies that they cannot measure the bedload discharge, which must therefore be measured with a different sampler or estimated with a bedload model.

The study of Cheng and Chiew (1998) presented a theoretical derivation for a new pickup probability formulation for sediment transport. Sand particles were assumed to be subjected to a hydraulically rough flow. The derived equation compares well with the experimental data found in published literature. Using the proposed relation, the Shields criterion for the definition of the threshold condition for sediment transport is equivalent to $0.6 \%$ of the pickup probability.

Results from five physical hydraulic model studies of riverside water intakes situated along the Missouri River reach between Sioux City, lowa, and St. Louis, Missouri were presented by Nakato and Ogden (1998). Movablebed, undistorted Froude-scale models were used to determine the effectiveness of structural modifications in the vicinity of the intake to limit the influx of bed-load sediments. Solutions developed in each case included a series of submerged flow-turning vanes located on the riverward side of the intake. A sediment-barrier wall between the vanes and intake increases the stream wise velocity component, enhancing the effectiveness of flowturning vanes in maintaining a deep scour trench. Effective solutions determined using physical hydraulic models were verified at the prototype scale, as demonstrated by years of trouble-free operation at locations where the recommended sediment-control measures have been installed. Results presented provided design guidelines for bed load sediment control at riverside water-intake structures on sand-bed rivers.

Although, a one-dimensional (1D) mathematical model based on the nonequilibrium transport of sediment is rational in principle, its application to rivers requires empirical determination of a key coefficient in the basic equation of transport, and this is known as the adjustment coefficient. It also requires, as all ID models do, empirical and rather arbitrary apportioning of the computed total scour or deposition to the wetted perimeter. An attempt was made by Zhou and Lin (1998) 
to avoid both. The equation of sediment transport was written for a vertical stream tube of infinitesimal width for which the adjustment coefficient has been rationally determined. A procedure of lateral integration was then applied to obtain the global values of the said coefficient for the entire cross section and the distribution of deposition/erosion to the wetted perimeter. The shape of the cross section is thus determined along with the amount of deposition/erosion. Results obtained with this model compare favorably with those of depth-integrated two-dimensional (2D) computations and physical model testing, both carried out for the dam neighborhood of the Three Gorges Project.

An optimal-control approach was presented by Zhu et al. (1999). It determines optimal locations and scheduling of dredging to minimize total cost and to obtain a channel where water depths are not less than specified values. The optimal-control problem is formulated and a method was developed. The optimization problem was solved by the conjugate gradient method, and the gradient of the cost function was calculated by solving the adjoint problem. A simulation study was conducted using a 2D numerical model to demonstrate the method.

Barkdoll and Ettema (1999) presented the findings of laboratory flume experiments conducted to determine the limits to which submerged vanes can be used in preventing excessive bed-sediment ingestion into lateral diversions of flow from alluvial channels. The experiments showed that a scheme of submerged vanes placed at the diversion entrance admits only a negligible rate of bed-sediment entry into the diversion when the ratio of unit discharge in the diversion to unit discharge in the main channel, $\mathrm{q}_{\mathrm{r}}$, is less than about 0.2 . Beyond this value, the effectiveness of the vanes diminishes. The sediment-control performance of the vanes can be enhanced in several ways. One enhancement is the use of a skimming wall in conjunction with the vanes. The wall and the vanes are effective for values of $\mathrm{q}_{\mathrm{r}}$ up to about 0.3. Another enhancement was to widen the diversion entrance such that, at the entrance, $\mathrm{q}_{\mathrm{r}}$ does not exceed about 0.3. Further potential enhancements (modified vane shape, uniformity of flow distribution into the diversion, and increased flow velocity into the diversion) were found not to be effective. The findings are supported by observations of flow and bed-sediment behavior at a flume-scale diversion.

Sumer et al. (2001) presented the results of an experimental investigation on suction removal of sediment from between armor blocks/stones placed on a loose bed. The process of suction has been investigated. It was found that the vortices that form in the holes between the armor blocks are key to the process. The sediment swept into these vortices is entrained into the main body of the flow by these same vortices (the suction removal of sediment from between the armor blocks). The critical condition for the onset of suction was determined. It was found that the onset of suction is governed by two parameters: (1) the Shields parameter (based on the sediment size); and (2) the ratio of sediment size to stone size, $d / D$.

The stability of randomly deposited sediment beds was examined by McEwan and Heald (2001) using a discrete particle model in which individual grains are represented by spheres. The results indicated that the threshold shear stress for flat beds consisting of cohesionless uniformly sized grains cannot be adequately described by a singlevalued parameter; rather, it is best represented by a distribution of values. Physically, this result stems from the localized heterogeneity in the arrangement of surface grains. For uniformly sized beds, geometric similarity exists such that the critical entrainment shear stress distributions scale directly with grain size. A Shields parameter of 0.06 is commonly used to define "threshold conditions," and it was found that this corresponds to a point on the distributions where approximately $1.4 \%$ by weight of the surface is mobile.

A simple theory was developed by Nino (2002) to account for the observed downstream variation of the median sediment size in Chilean rivers based on a reachwise equilibrium sediment transport concept. The theory makes use of a bedload transport equation linked with a resistance equation to estimate the median sediment diameter as a function of channel slope, with the flow Reynolds number and bedload concentration as parameters. Both, Meyer-Peter and Mu"ller's and Ackers and White's formulas are used alternatively as bedload equations. A Manning-Strickler type of formulation was used as a resistance relationship. The resulting model was validated against field data corresponding to 150 rivers in Central Chile, covering slopes in the range of 0.04 to $8.61 \%$, with median sediment size in the range of 0.3 to $250 \mathrm{~mm}$. Despite the simplicity of the theory and the somewhat bold assumptions made in its derivation, the estimated variation of the median sediment size with channel slope follows the same trend as the field data. Most of the scatter of these data falls within the theoretical limits given by the estimated range of values of the parameters of the model.

Experimental results on sediment removal efficiency of vortex chamber type sediment extractors were reported by Athar et al. (2002). A geometric configuration of the extractor was identified that is able to remove even the fine sediment $(0.055<\mathrm{d}<0.22 \mathrm{~mm})$ from flow with high efficiency. For the purpose of analysis, the experimental data of present study were compiled with the data from other laboratories and the field that were collected previously by other investigators. Since the existing relations were not found to produce satisfactory results, a new relationship was developed for determination of the sediment removal efficiency of the vortex chamber type sediment extractors.

A steady, two-dimensional numerical model was created by Naser et al. (2005) to study the hydrodynamics of a rectangular sedimentation basin 




a

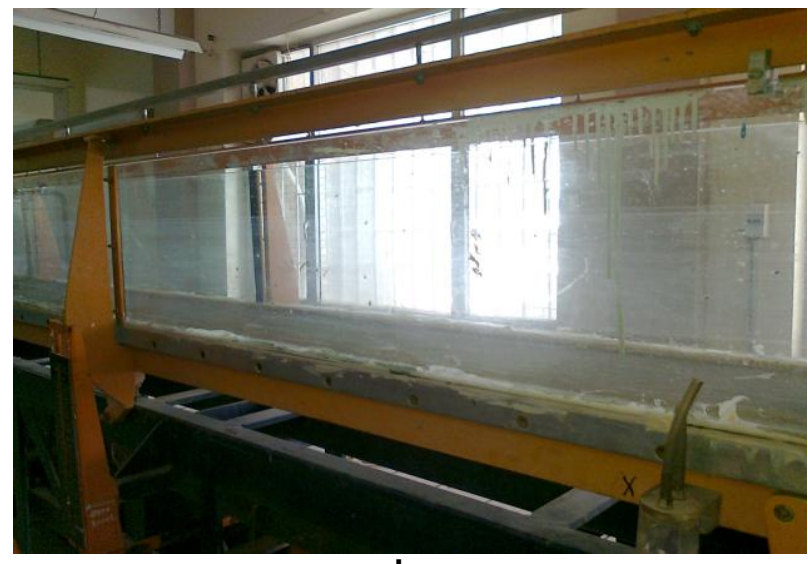

b

Figure 1. Open channel used in the experiments.

under turbulent conditions. The strip integral method was used to formulate the flow equations, using a forward marching scheme for solving the governing partial differential equations of continuity, momentum, advection-diffusion, turbulent kinetic energy, and its dissipation. In this way the flow equations were converted to a set of ordinary differential equations in terms of the key physical parameters. These parameters, along with a set of shape functions, describe flow variables including the velocity, the concentration of suspended sediments, and both the kinetic energy and its dissipation rate. Four Gaussian distributions were investigated, one corresponding to each flow parameter. In order to calculate the turbulent shear stresses, a two-equation turbulence model (that is, $k-\varepsilon$ model) was used. A fourth order Runge-Kutta method numerically integrates the set of ODEs. Simulation results were compared with experimental data, and close agreement (generally within 5 to $10 \%$ ) was observed.

Gopakumar and Jesuraj (2012) dealt with development of a new mathematical model to simulate non-equilibrium transport of suspended sediment in open channels, with special emphasis on sediment overloading. Equilibrium transport of bed sediment was also included. Sources of the suspended sediment were from the catchment (such as construction sites, mining areas etc.). This sediment was brought into rivers and canals by flowing rainwater.

Its overloading can result in large changes in bed and water levels of the rivers and also in heavy silting of the canal beds. A new mathematical model to simulate this effect was derived based on the control volume approach. The model was tested using data available in literature and results were found satisfactory. The developed model was then applied to a hypothetical flood and sediment routing problem in a river and analyses of the results were given.

The objective of the present study is to build a small scale open channel model and study the effect of some parameters on sedimentation. These parameters include channel width, bed slope and flow discharge.

\section{Laboratory works}

In the present work, eighty-one experiments on the laboratory open-channel of cross-section dimensions $(20 * 50) \mathrm{cm}$ and (10) $\mathrm{m}$ long as shown in Figure 1. Each test was repeated three times and the average value for sediment concentration was calculated. The experiments included using sharp crested weir in the last third part of the channel. Three heights of weir were used; namely half width of channel $(\mathrm{B})$, equal to the width, and 1.5 times the width of channel. Also, three bed slopes of the channel are used; namely $0.015,0.0225$, and 0.03 .

In all experiments, the values of discharge are limited between 100 and $300 \mathrm{l} / \mathrm{min}$. The concentration of suspended sediment was measured upstream and downstream the weir through each experiment to calculate the sediment reduction ratio (SRR\%) as follows:

$S R R \%=\frac{S_{u s}-S_{d s}}{S_{u s}} * 100-----$

where: $S_{\text {us }}=$ Suspended sediment concentration upstream weir, and $S_{d s}=$ Suspended sediment concentration downstream weir.

The water used in the experiments was taken from the Euphrates river at Al-Ramadi city west of Iraq.

\section{ANALYSIS OF RESULTS AND DISCUSSION}

Three variables are studied in this research which are the weir height, the slope of channel bed, and the discharge. To study their effect on the efficiency of weir in sediment 


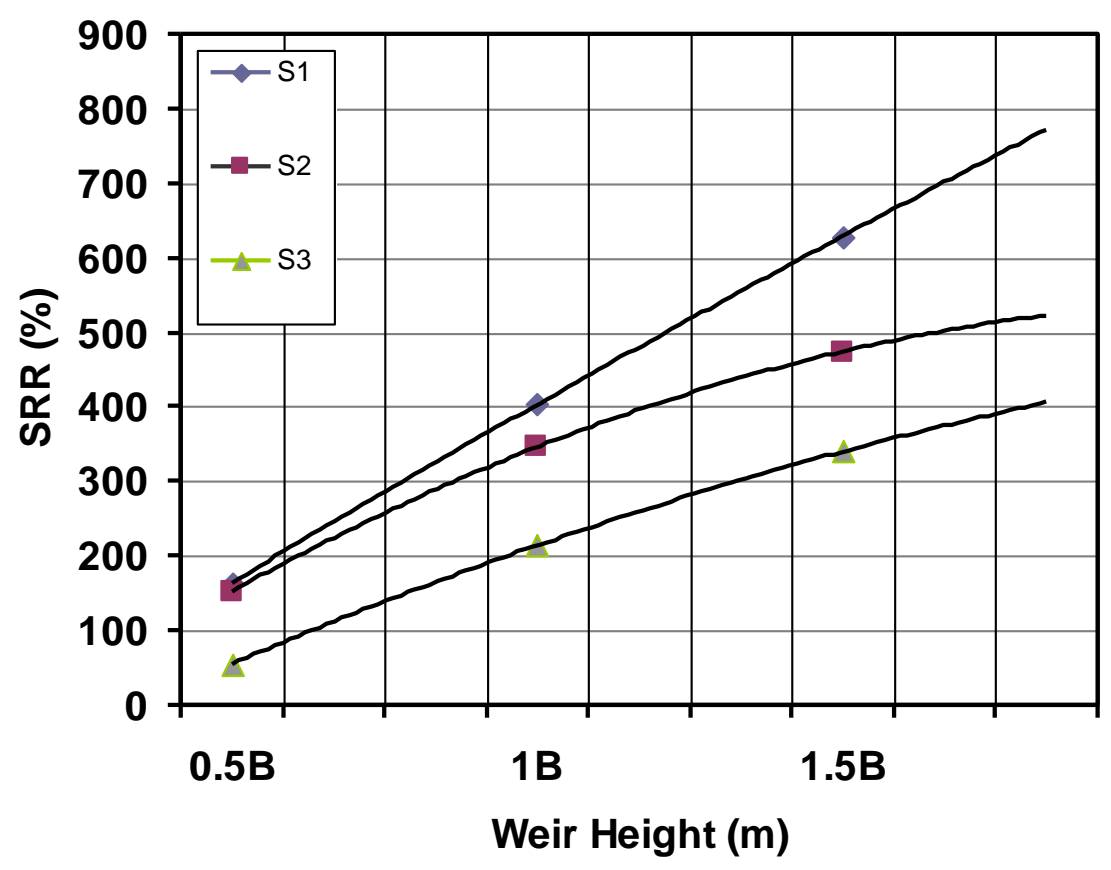

Figure 2. Variation of sediment reduction ratio with weir height for different slopes of the channel, discharge $=100 \mathrm{l} / \mathrm{min}$.

removal, the sediment reduction ratio (SRR\%) is measured. It was noted that (SRR\%) increases with the increase of weir height, but decreases while the channel bed slope increases as shown in Figure 2. The figure shows that (SRR\%) reaches about (63\%) at channel bed slope $(0.015)$ and weir height $(1.5 B)$ where $(B)$ is the width of channel.

The results coincide with the principles of hydraulics of flow which state that with increasing the slope of the channel bed, the flow velocity increases which causes to lift the particles through water depth and reduce the probability of sediment trapping. The figure shows also that $(\mathrm{SRR} \%)$ increases with increasing of the weir height due to the same reason.

The results for other values of discharge revealed the same relations shown in Figure 2 for discharge (100 $1 / \mathrm{min}$ ) and these results are illustrated in Figures 3 and 4 for a discharge value of 200 and $300 \mathrm{l} / \mathrm{min}$, respectively.

Figures 5, 6 and 7 show the variation of (SRR\%) with the discharge for three channel bed slopes and weir heights. It is noted that when the discharge is greater than $(100 \mathrm{l} / \mathrm{min})$, the highest values of (SRR\%) are obtained for the three slopes and at weir height (1.5B) as shown in Figure 5 . The figures also show that the differences between the values of (SRR\%) for discharges $(100 \mathrm{l} / \mathrm{min})$ and $(200 \mathrm{l} / \mathrm{min})$ are not significant, then the $(300 \mathrm{l} / \mathrm{min}$ ) is the discharge suitable to provide acceptable (SRR\%).

This can be attributed to that the initial motion of a sediment particle occurs when the ratio of the hydrodynamic force to the submerged weight force acting on the particle exceeds a certain limit determined by the geometry of the particle and the particle Reynolds number.

In Figure 8 , the variation of sediment reduction ratio is drawn with discharge for different heights of weir. The figure shows that (SSR\%) increases slightly with the increase of discharge. For the first slope $(0.015)$, the increasing in discharge causes a little increase in flow velocity and the difference is converted into cross section flow area. Then, the difference in (SRR\%) for the two values of discharge; 200 and $300 \mathrm{l} / \mathrm{min}$ is slightly small, the value of discharge of $300 \mathrm{l} / \mathrm{min}$ is suitable to give considerable (SRR\%) for all weir heights. The role of fluid discharge approves that the sedimentation process must consider both the characteristics of the sediment and the fluid motion as was stated by Naser et al. (2005).

The same trend of variation of (SRR\%) with discharge is observed in Figures 9 and 10, but the values of SRR decrease with the increase of the channel bed slope because the increase of bed slope leads to increase the flow velocity more than the cross sectional area of flow.

This behavior can be understood since the relevant sediment characteristics are not only the settling velocities of the individual particles but also whether they retain their individualities as they settle. When two particles of a nonflocculent material come together, the only effect is the momentary mutual interference; if two particles of flocculent material interact they combine into a new particle with different properties. Thus, during the sedimentation of nonflocculent materials, the sediment characteristics are constant, while the characteristics of 


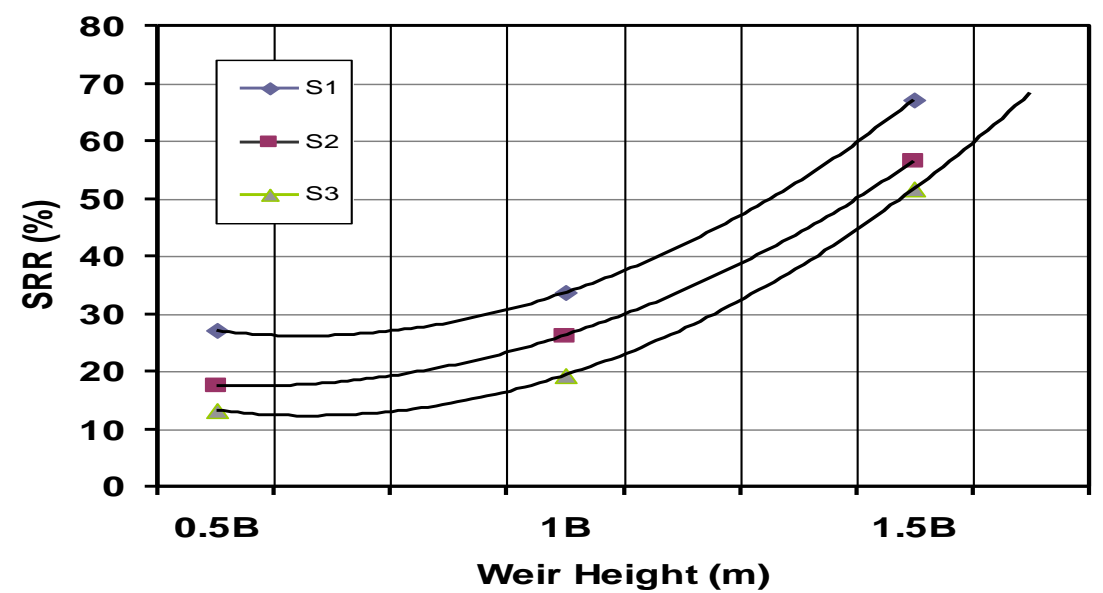

Figure 3. Variation of sediment reduction ratio with weir height for different slopes of the channel, discharge $=200 \mathrm{l} / \mathrm{min}$.

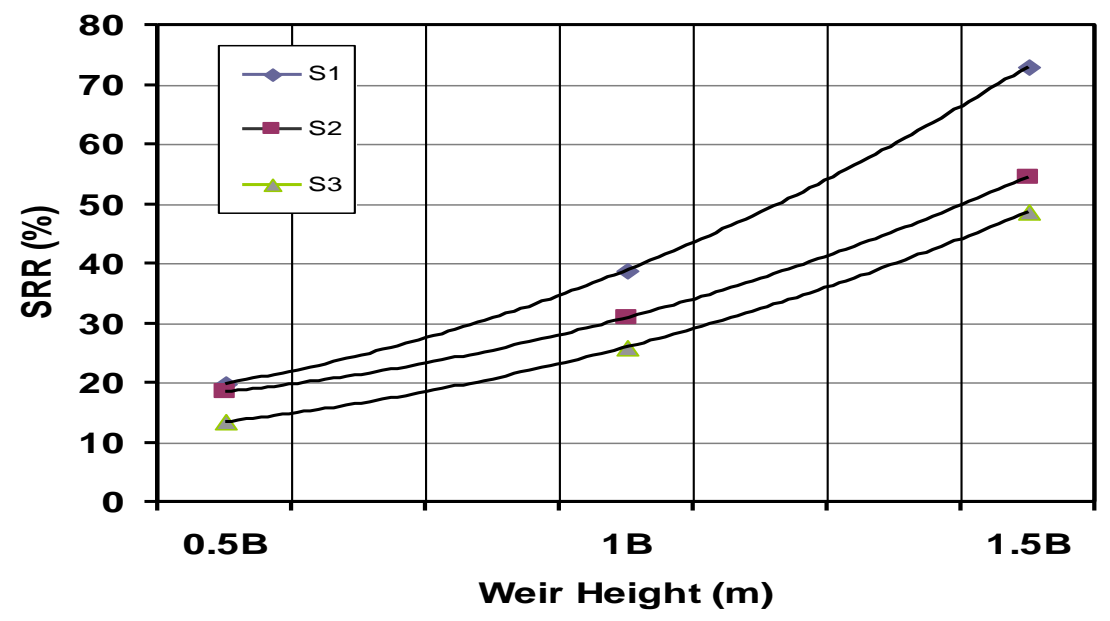

Figure 4. Variation of sediment reduction ratio with weir height for different slopes of the channel, discharge $=300 \mathrm{l} / \mathrm{min}$.



Figure 5. Variation of sediment reduction ratio with discharge for different slopes of the channel, $\mathrm{H}=0.5 \mathrm{~B}$. 


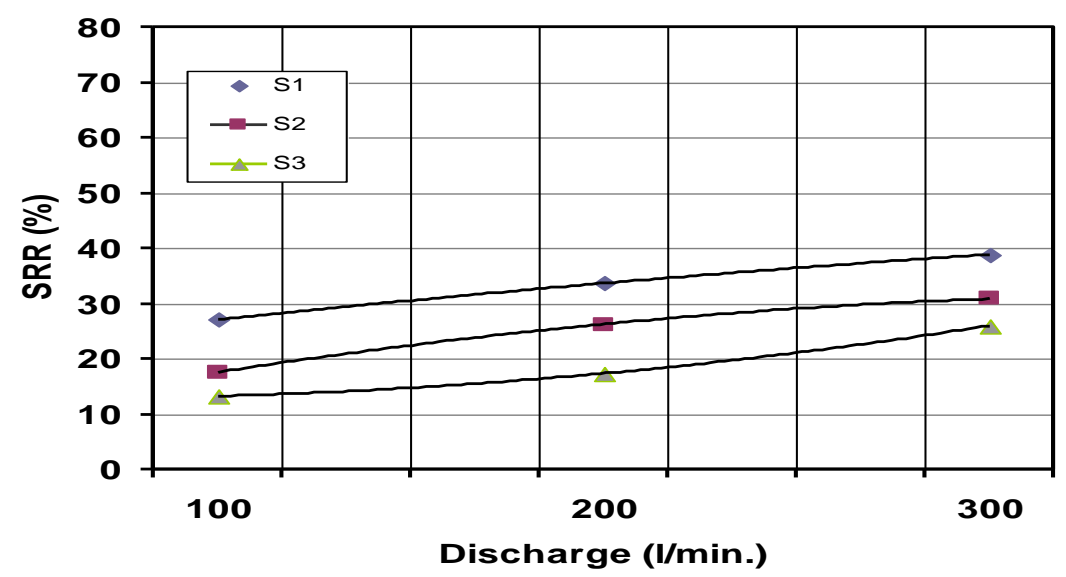

Figure 6. Variation of sediment reduction ratio with discharge for different slopes of the channel, $\mathrm{H}=\mathrm{B}$.

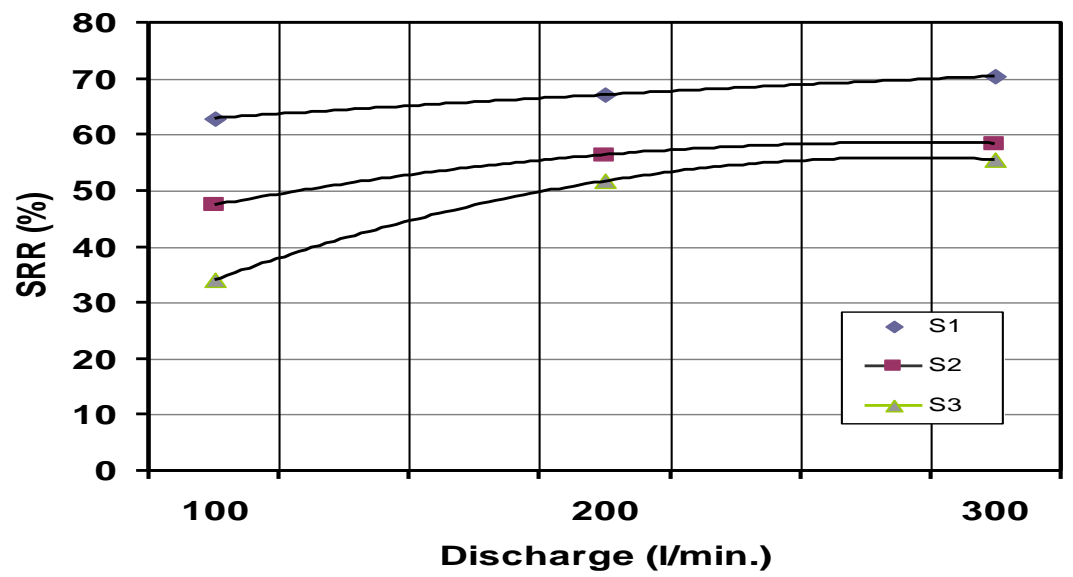

Figure 7. Variation of sediment reduction ratio with discharge for different slopes of the channel, $\mathrm{H}=1.5 \mathrm{~B}$.

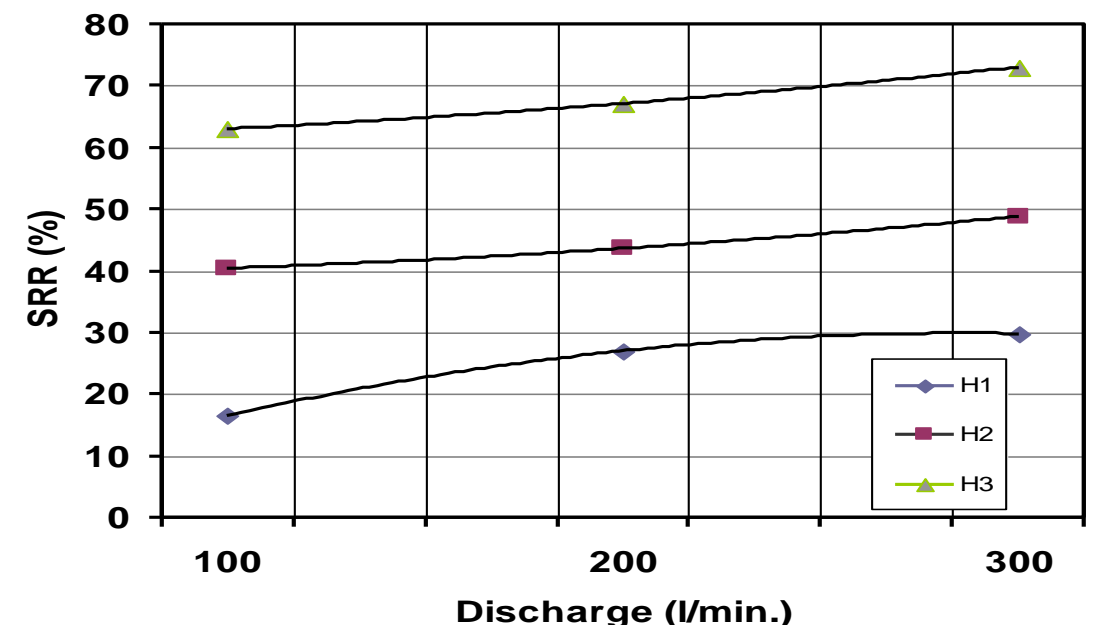

Figure 8. Variation of sediment reduction ratio with discharge for weir heights of the channel, slope $S_{1}$. 


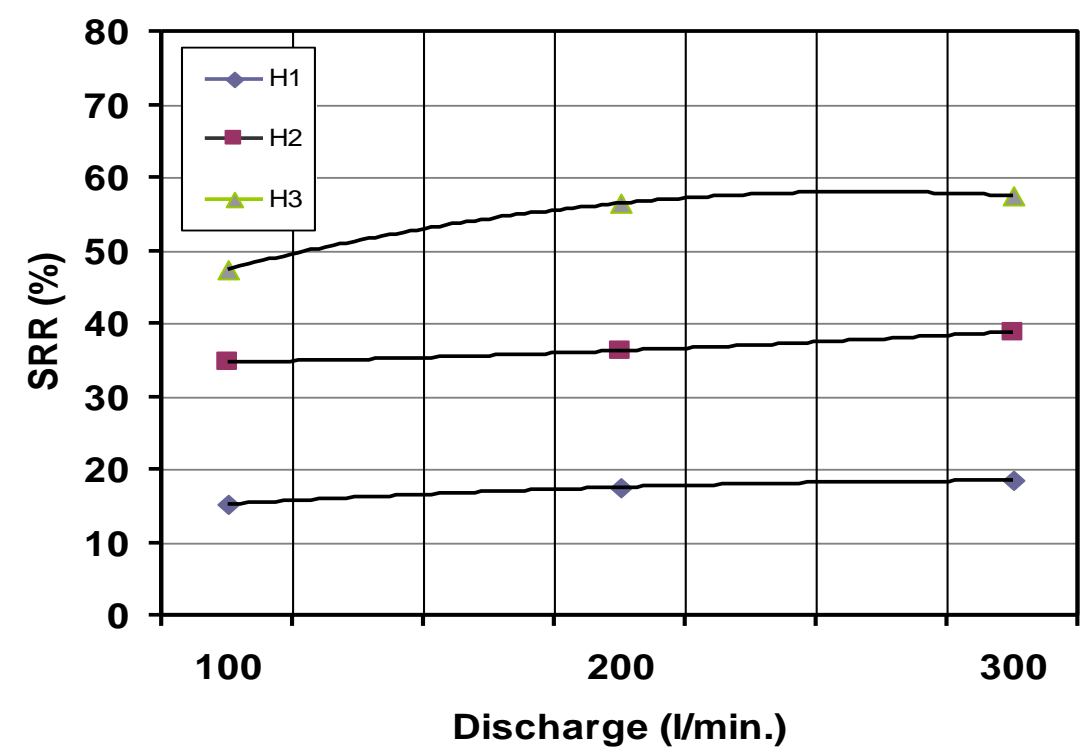

Figure 9. Variation of sediment reduction ratio with discharge for weir heights of the channel, slope $\mathrm{S}_{2}$.

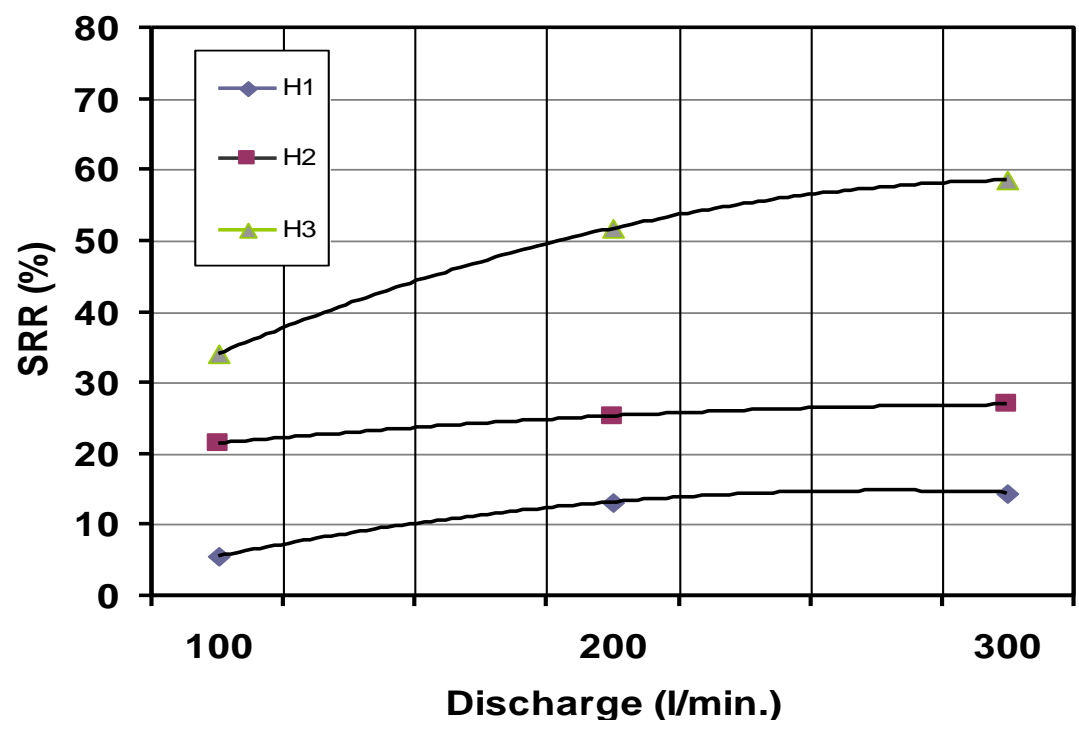

Figure 10. Variation of sediment reduction ratio with discharge for weir heights of the channel, slope $S_{3}$.

flocculent materials change continuously in a way that depends on both the sediment and flow characteristics (Naser et al., 2005).

\section{Conclusions}

Three variables were studied in this research which are the weir height, the slope of channel bed, and the discharge. Studying their effect on the efficiency removal of sediments leads to the following conclusions:

1. The sediment reduction ratio increases with the increase of weir height and reaches about $(63 \%)$ at a channel bed slope of $(0.015)$ and a weir height of $1.5 \mathrm{~B}$ (where $B$ is the width of channel).

2. When the discharge is more than $(100 \mathrm{l} / \mathrm{min})$, the highest values of sediment reduction ratio are obtained 
for the three slopes used and at a weir height of 1.5B. 3. The value of discharge $(300 \mathrm{l} / \mathrm{min})$ is suitable to provide a considerable sediment reduction ratio for all weir heights.

\section{Conflict of Interests}

The author(s) have not declared any conflict of interests.

\section{REFERENCES}

Athar M, Kothyari UC, Garde RJ (2002). Sediment Removal Efficiency of Vortex Chamber Type Sediment Extractor, J. Hydraulic Eng, ASCE, 128(12):1051-1059.

Barkdoll BD, Ettema R (1999). Sediment Control at Lateral Diversions: Limits and Enhancements to Vane Use, J. Hydraulic Eng. ASCE, 125(8):862-870.

Cheng NS, Chiew YM (1998). Pickup Probability for Sediment Entrainment, J. Hydraulic Eng. ASCE, 124(2):232-235.

Gopakumar R, Jesuraj R (2012). Simulation of Non-Equilibrium Transport of Suspended Sediment in Open Channels, Int. J. Soft Comput. Eng. (IJSCE), 2(5):57-61.

Lyn DA (2003). Sediment Transport in Open Channels, in the "Civil Engineering Handbook", edited by WF CHEN, JY. Richard, Lie W, second edition, 2003, CRC PRESS.

McEwan I, Heald J (2001). Discrete Particle Modeling of Entrainment from Flat Uniformly Sized Sediment Beds, J. Hydraulic Eng. ASCE, 127(7):588-597.

Nakato T, Ogden FL (1998). Sediment Control at Water Intakes along Sand-Bed Rivers, J. Hydr. Eng. ASCE, 124(6):589-5996.
Naser Gh, Karney BW, Salehi AA (2005). Two-Dimensional Simulation Model of Sediment Removal and Flow in Rectangular Sedimentation Basin, J. Environ. Eng. ASCE, 131(12):1740-1749.

Nino Y (2002). Simple Model for Downstream Variation of Median Sediment Size in Chilean Rivers, J. Hydr. Eng. ASCE, 128(10):934941.

Sumer BM, Cokgor S, Fredsøe J (2001). Suction Removal of Sediment from Between Armor Blocks, J. Hydr. Eng. ASCE, 127(4):293-306.

Zhou J, Lin B (1998). One -Dimensional Mathematical Model for Suspended Sediment by Lateral Int. J. Hydraulic Eng. ASCE, 124(7):712-717.

Zhu J, Zeng Q, Guo D, Liu Z (1999). "Optimal Control of Sedimentation in Navigation Channels, J. Hydr. Eng. ASCE, 125(7):750-759. 